\title{
Anisotropic thermal Sunyaev-Zel'dovich effect and the possibility of an independent measurement of the CMB dipole, quadrupole and octupole
}

\author{
I.G. Edigaryev, D.I. Novikov, and S.V. Pilipenko \\ Astro-Space Center of P.N. Lebedev Physical Institute, Profsoyusnaya 84/32, Moscow, Russia 117997.
}

\begin{abstract}
We consider the effect of the cosmic microwave background (CMB) frequency spectral distortions arising due to the Compton scattering of the anisotropic radiation on Sunyaev-Zel'dovich (SZ) clusters. We derive the correction to the thermal SZ effect due to the presence of multipoles with $\ell=1,2,3$ in the anisotropy of the CMB radiation. We show that this effect gives us an opportunity for an independent evaluation of the CMB dipole, quadrupole and octupole angular anisotropy in our location using distorted signal from the nearby galaxy clusters and to distinguish between the SachsWolfe (SW) and the Integrated SachsWolfe (ISW) effects by combining such signals from distant and nearby clusters. The future space mission 'Millimetron' will have unprecedented sensitivity, which will make it possible to observe the spectral distortion we are considering.

PACS numbers:

Keywords: Cosmic Microwave Background, spectral distortions, SZ effect, cosmology
\end{abstract}

\section{INTRODUCTION}

In this paper we propose a method for the independent estimation of the Cosmic Microwave Background (CMB) dipole, quadrupole and octupole anisotropy using measurements of the relic radiation frequency spectrum distortion from nearby and distant Sunyaev-Zel'dovich clusters.

In the past two decades, a large number of papers, devoted to the theoretical study of various corrections to the Kompaneets equation 1 and the Sunyaev-Zel'dovich effect [2, 3] have been published. These studies take into account relativistic corrections to the thermal SZ effect [4] and multiple scattering on SZ clusters [8]. Recently in Ref. 9] the temperatures of galaxy clusters were measured for the first time using the relativistic corrections to the thermal SZ effect only. The relativistic correction approach was adopted in Refs. [10 12 for moving clusters of galaxies to find corresponding corrections for the kinematic SZ effect. In Ref. [13] the covariant formalism for the polarization SZ effect was applied. In Refs. [14] a way to cleanly separate kinematic and scattering terms was obtained. In Ref. [15 17, a detailed analytic investigation of the Boltzmann equations was made for three Lorentz frames and expressions for the photon redistribution functions were derived. As for the study of the intensity distortions, the basic assumption of these works is the CMB is isotropic.

The influence of the CMB anisotropy on the spectral distortions was investigated in Refs. [18, 19] for the kinetic Sunyaev-Zel'dovich effect. In that work polarization and intensity distortions in moving clusters were considered, but for simplicity the thermal effect was not taken into account. In Ref. [20] it was demonstrated how spectral measurements of the low multipoles can be used to separate the motion-induced dipole of the CMB from a possible intrinsic dipole component.

The generalized Kompaneets equation was considered in Ref. $21-23$.
In our paper we consider the intensity spectral distortion due to Compton scattering of anisotropic CMB radiation on nonmoving galaxy clusters. We do not take into account the movement of clusters since the effect under consideration can be easily separated from the effects associated with clusters' peculiar velocities.

Compton scattering of an anisotropic blackbody radiation on plasma causes a very characteristic spectral distortion due to the presence of multipoles with $\ell=1,2,3$ in the radiation anisotropy. This effect is the anisotropic correction to the classical thermal SZ effect and follows directly from the anisotropic Kompaneets equation first derived in Ref. [24] in linear approximation in $k T_{e} / m_{e} c^{2}$. The amplitude of this distortion depends on the powers of $C_{1}, C_{2}, C_{3}$ multipoles and their orientation with respect to the axis connecting the scattering point and the observer. We will show, that the shape of this distortion is very characteristic and allows to distinguish it from other nonblackbody components.

The CMB radiation for the observer located at a nearby SZ cluster has roughly the same anisotropy map as the radiation that we directly observe in the sky including the quadrupole and octupole. We will show, that for redshifts $z \sim 0.05$ the variations of multipole amplitudes are about 10\%. Therefore, the measurements of the spectral distortions from such clusters caused by the presence of dipole, quadrupole and octupole anisotropy at the moment of scattering gives us an opportunity for an independent estimation of the $\ell=1,2,3$ CMB multipoles and their orientations. According to WMAP and Planck results, the low CMB anisotropy multipoles have insufficient power (compare to the expected one) [25-28. Besides there is a quadrupole-octupole alignment [29 31]. These facts are rather difficult to explain in the framework of Gaussian statistics. As for the CMB intrinsic dipole it is completely overshadowed by our motion with respect to the CMB frame. Thus, an independent estimate of the powers of quadrupole and octupole (our local multipoles we directly observe by WMAP and Planck) and their orientations can be quite important. Besides it 
can provide us with the information about the intrinsic dipole in our location.

At the same time, the use of such a signal from distant clusters can provide us with unique information about the pure SachsWolfe (SW) effect without the integrated part of it which is the Rees-Sciama (RS) [32] or Integrated Sachs-Wolfe (ISW) effect. The ISW effect creates its contribution to the CMB low multipoles at very small redshifts. Therefore the probe of the spectral distortions caused by the anisotropic SZ effect from high redshift SZ clusters is ISW-free.

The upcoming Millimetron mission 33 35 will have unprecedented sensitivity $(\sim 1 \mathrm{Jy} / \mathrm{Sr})$ in the single dish mode to measure the signal in the range from $60 \mathrm{GHz}$ to $1 \mathrm{THz}$. One of the main tasks of this mission will be the measurement of so called $\mu$ - and $y$ - spectral distortions of $\mathrm{CMB}$ radiation, which arise due to the energy injections into the plasma in the prerecombination epoch (see for instance Refs. [36, 37]). As an additional task, the possibility of detecting the spectral distortions from SZ clusters caused by the presence of CMB anisotropy can be considered.

As it was mentioned above, in the approximation in $k T_{e} / m_{e} c^{2}$ only the first three multipoles of the CMB anisotropy (dipole, quadrupole and octupole) form the additional spectral distortion in the thermal SZ effect. In order to separate the signal, caused by the presence of these multipoles at the moment of scattering from other spectral distortions it is necessary to take into account all the effects of higher or the same order for the isotropic (monopole) CMB fraction. These effects include relativistic corrections to the thermal SZ effect at least up to the fifth order in $k T_{e} / m c^{2}$, corrections to the kinetic SZ effect and the effect of multiple scattering.

The paper is organized as follows: In Section II we review the equation for the radiative transfer in plasma for relatively cold electrons and photons for the linear order in $h \nu / m_{e} c^{2}, k T_{e} / m_{e} c^{2}$. Using this equation we derive the correction to the thermal SZ effect due to the presence of dipole, quadrupole and octupole anisotropies in $\mathrm{CMB}$ radiation. In Section III we present the method for independently estimating the $\ell=1,2,3$ multipoles in our location using nearby SZ clusters. In this Section we also show how to distinguish between SW and ISW effects combining the signals from nearby and distant clusters. Finally in Section IV we make our conclusions.

\section{THE ANISOTROPIC KOMPANEETS EQUATION AND THE ANISOTROPIC THERMAL SZ EFFECT}

In this Section we review the anisotropic Kompaneets equation found by Babuel-Peyrissac and G. Rouvillois [24] and derive the formula for the anisotropic thermal SZ effect.

We use the following notations:
$I=I(\nu):$ spectral radiance;

$n(\nu, \boldsymbol{\Omega})=\frac{c^{2} I}{2 h \nu^{3}}$ : photon concentration in phase space (photon number density), where $\nu, c, h$ are the frequency, the speed of light and the Planck constant respectively;

$\boldsymbol{\Omega}^{\prime}, \boldsymbol{\Omega}$ : radiation propagation directions before and after scattering;

$\mu=\Omega \Omega^{\prime}:$ cosine of the scattering angle;

$T_{e}, T_{r}$ : temperatures of electrons and radiation;

$m_{e}$ : electron mass at rest;

$N_{e}$ : concentration of electrons;

$\Theta_{e}=\frac{k T_{e}}{m_{e} c^{2}}$, where $k$ is the Boltzmann constant;

$\sigma_{T}$ : Thomson cross section;

we also use the operator $\frac{D}{D \tau}=\frac{1}{N_{e} \sigma_{T}} \frac{d}{c d t}+\Omega \nabla$ and the variable $x=\frac{h \nu}{k T_{r}}$.

The equation for the radiative transfer in plasma was derived in Ref. 24] for relatively cold photons and electrons: $k T_{e} \ll m_{e} c^{2}, h \nu \ll m_{e} c^{2}$. This approximation is quite correct for the typical SZ clusters with $T_{e} \sim 10 \mathrm{KeV}$ and relic photons with $T_{r} \sim 3^{\circ} \mathrm{K}$. The equation we consider, describes the correction to the Thomson scattering up to the first order in $h \nu / m_{e} c^{2}$, $k T_{e} / m_{e} c^{2}$.

In order to separate the anisotropic and isotropic parts of radiation we denote by $\Delta$ the difference between the photon number density before and after scattering: $\Delta=$ $n\left(x, \boldsymbol{\Omega}^{\prime}\right)-n(x, \boldsymbol{\Omega})$. Following Ref. [24] and using our notations one can rewrite the equation for the radiative transfer in plasma in terms of $n(x, \boldsymbol{\Omega})$ :

$$
\begin{aligned}
& \frac{D n}{D \tau}=\frac{3}{16 \pi} \int_{\Omega^{\prime}}\left\{\left(1+\mu^{2}\right) \Delta+\right. \\
& +\Theta_{e}\left(4 \mu^{3}-6 \mu^{2}-4 \mu+2-2 \frac{T_{r}}{T_{e}} x\left(1+\mu^{2}\right)(1-\mu)\right) \Delta+ \\
& +\Theta_{e}\left(\frac{1}{x^{2}} \frac{\partial}{\partial x}\left[x^{4}\left(\frac{T_{r}}{T_{e}} \Delta+\frac{\partial \Delta}{\partial x}\right)\right]+2 \frac{T_{r}}{T_{e}} n \frac{\partial}{\partial x}\left[x^{2} \Delta\right]\right) . \\
& \left.\cdot\left(1+\mu^{2}\right)(1-\mu)\right\} d \Omega^{\prime}+ \\
& +\Theta_{e} \frac{1}{x^{2}} \frac{\partial}{\partial x}\left[x^{4}\left(\frac{T_{r}}{T_{e}}\left(n+n^{2}\right)+\frac{\partial n}{\partial x}\right)\right]+O\left(\Theta_{e}^{2}\right),
\end{aligned}
$$

where $n=n(x, \boldsymbol{\Omega})$. The integral part of this equation represents the contribution from the anisotropic radiation, while the last term gives the usual right hand side of the Kompaneets equation. Taking into account that $T_{r} / T_{e}$ is extremely small for SZ clusters, we neglect all 
terms proportional to this ratio:

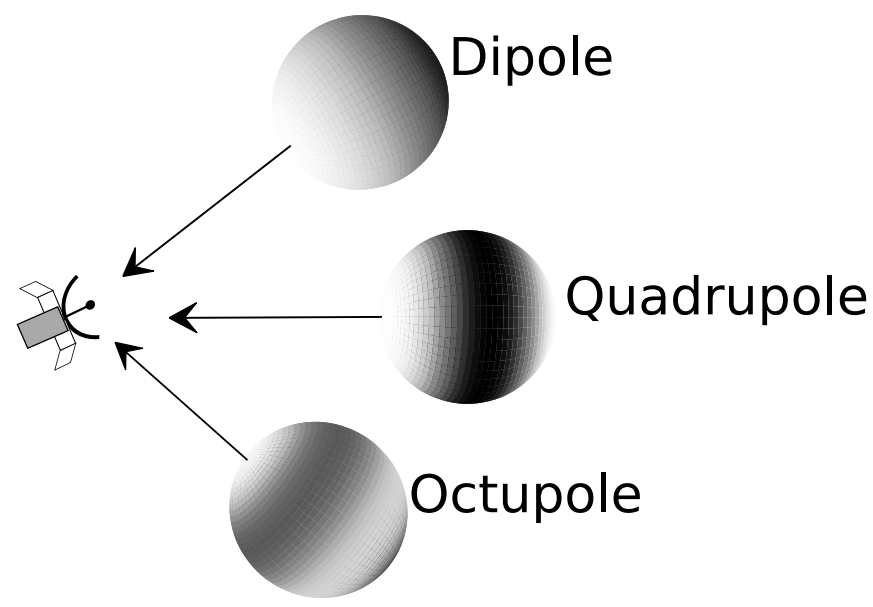

FIG. 1: Schematic representation of multipole components which affect the observed CMB spectrum due to scattering. Only components with $\ell=1,2,3$ and with the symmetry axis directed towards an observer contribute to the spectral distortion.

$$
\begin{aligned}
& \frac{D n}{D \tau}=\frac{3}{16 \pi} \int_{\boldsymbol{\Omega}^{\prime}}\left(1+\mu^{2}\right) \Delta d \boldsymbol{\Omega}^{\prime}+ \\
& +\Theta_{e} \frac{3}{16 \pi} \int_{\boldsymbol{\Omega}^{\prime}}\left\{\left(4 \mu^{3}-6 \mu^{2}-4 \mu+2\right) \Delta+\right. \\
& \left.\frac{1}{x^{2}} \frac{\partial}{\partial x}\left[x^{4} \frac{\partial}{\partial x}(n+\Delta)\right]\left(1+\mu^{2}\right)(1-\mu)\right\} d \boldsymbol{\Omega}^{\prime}
\end{aligned}
$$

The cosmic microwave background radiation has a spectrum that coincides with great accuracy with the blackbody spectrum, defined by the temperature $T_{r}$. Since the relic radiation is anisotropic, its temperature varies with direction. Thus, for radiation incident from the direction $\boldsymbol{\Omega}^{\prime}$, we can write the following formula:

$$
\begin{aligned}
& n\left(x, \boldsymbol{\Omega}^{\prime}\right)=B+\frac{d B}{d x} \frac{d x}{d T_{r}} \Delta_{T}\left(\boldsymbol{\Omega}^{\prime}\right) \\
& B(x)=\frac{1}{e^{x}-1}
\end{aligned}
$$

where $\Delta_{T}\left(\boldsymbol{\Omega}^{\prime}\right)=T\left(\boldsymbol{\Omega}^{\prime}\right)-T_{r}$. Therefore, in order to find the spectral distortions that arise during a single scattering of photons on plasma in SZ clusters, we substitute the following initial conditions into Eq. (2):

$$
\begin{aligned}
& n\left(x, \boldsymbol{\Omega}^{\prime}\right)=n+\Delta=B-x \frac{d B}{d x} \frac{\Delta_{T}\left(\boldsymbol{\Omega}^{\prime}\right)}{T_{r}}, \\
& \Delta=-x \frac{d B}{d x}\left(\frac{\Delta_{T}\left(\boldsymbol{\Omega}^{\prime}\right)}{T_{r}}-\frac{\Delta_{T}(\boldsymbol{\Omega})}{T_{r}}\right)
\end{aligned}
$$

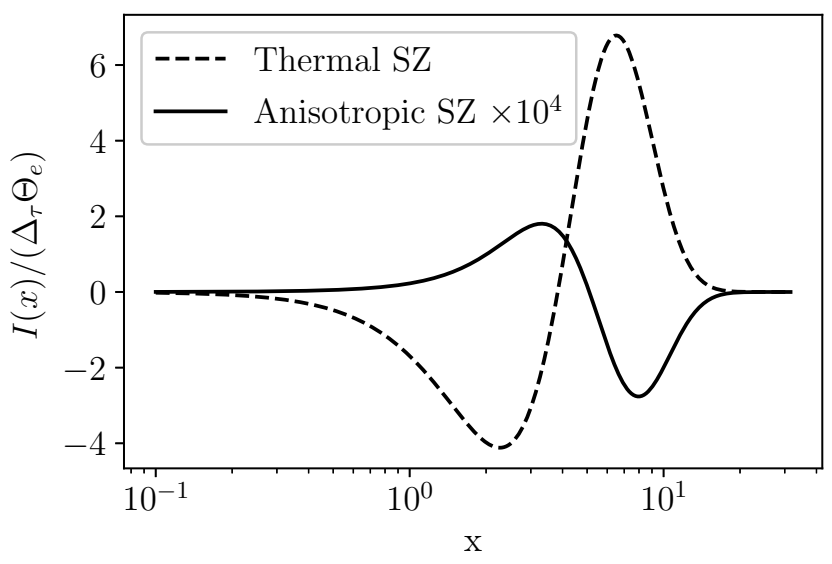

FIG. 2: The comparison of the classical thermal SZ effect (dashed line) with the spectral distortion caused by the presence of $\ell=1,2,3$ anisotropy multipoles (Anisotropic thermal SZ effect) (solid line).

Finally we get the result for the changes in photon number density $\Delta_{n}$ in the optically thin limit:

$$
\begin{aligned}
& \Delta_{n} / \Delta_{\tau}= \\
& \left.=\left(\beta_{1}+\Theta_{e} \beta_{2}\right)\left(-x \frac{d B}{d x}\right)+\quad \quad\right\} 1 \\
& \left.+\Theta_{e} \beta_{3} \frac{1}{x^{2}} \frac{d}{d x}\left[x^{4} \frac{d}{d x}\left(-x \frac{d B}{d x}\right)\right]+\quad\right\} 2 \\
& \left.+\Theta_{e} \frac{1}{x^{2}} \frac{d}{d x}\left[x^{4} \frac{d B}{d x}\right], \quad \quad\right\} t S Z
\end{aligned}
$$

where $\Delta_{\tau}$ is the optical depth. The first two terms in Eq. (5) are due to the presence of non-zero CMB anisotropy. The physical meaning of these terms is given below. The third term represents the classical thermal SZ effect for the isotropic fraction of radiation. According to Eq. (2) expressions for the coefficients $\beta_{i}$ are as follows:

$$
\begin{aligned}
& \beta_{1}=\frac{3}{16 \pi} \int_{\boldsymbol{\Omega}^{\prime}}\left(1+\mu^{2}\right)\left(\frac{\Delta_{T}\left(\boldsymbol{\Omega}^{\prime}\right)}{T_{r}}-\frac{\Delta_{T}(\boldsymbol{\Omega})}{T_{r}}\right) d \boldsymbol{\Omega}^{\prime}, \\
& \beta_{2}=\frac{3}{16 \pi} \int_{\boldsymbol{\Omega}^{\prime}}\left(4 \mu^{3}-6 \mu^{2}-4 \mu+2\right)\left(\frac{\Delta_{T}\left(\boldsymbol{\Omega}^{\prime}\right)}{T_{r}}-\frac{\Delta_{T}(\boldsymbol{\Omega})}{T_{r}}\right) d \boldsymbol{\Omega}^{\prime}, \\
& \beta_{3}=\frac{3}{16 \pi} \int_{\boldsymbol{\Omega}^{\prime}}(1-\mu)\left(1+\mu^{2}\right) \frac{\Delta_{T}\left(\boldsymbol{\Omega}^{\prime}\right)}{T_{r}} d \boldsymbol{\Omega}^{\prime} .
\end{aligned}
$$

For the observer located at the SZ cluster the CMB radiation anisotropy can be described in terms of spherical harmonics $Y_{\ell}^{m}\left(\boldsymbol{\Omega}^{\prime}\right)$ : 


$$
\frac{\Delta_{T}\left(\boldsymbol{\Omega}^{\prime}\right)}{T_{r}}=\sum_{\ell=1}^{\infty} \sum_{m=-\ell}^{\ell} \tilde{a}_{\ell m} Y_{\ell}^{m}\left(\boldsymbol{\Omega}^{\prime}\right),
$$

where $\tilde{a}_{\ell m}$ are the coefficients for $\Delta T / T$ decomposition for a given location of the galaxy cluster. If we choose a local spherical coordinate system at the scattering point in such a way that the north pole points to the observer [which is the same as the direction $\boldsymbol{\Omega}$ in Eqs. (1) and (2)], then in terms of such a system the coefficients $\beta_{i}$ are as follows:

$$
\begin{aligned}
& \beta_{1}=\frac{1}{4 \sqrt{\pi}}\left(\frac{1}{\sqrt{5}} \tilde{a}_{20}-2 \frac{\Delta_{T}(\boldsymbol{\Omega})}{T_{r}}\right) \\
& \beta_{2}=-\frac{3}{2 \sqrt{\pi}}\left(\frac{2}{5 \sqrt{3}} \tilde{a}_{10}+\frac{1}{\sqrt{5}} \tilde{a}_{20}-\frac{2}{5 \sqrt{7}} \tilde{a}_{30}\right), \\
& \beta_{3}=-\frac{3}{4 \sqrt{\pi}}\left(\frac{4}{5 \sqrt{3}} \tilde{a}_{10}-\frac{1}{3 \sqrt{5}} \tilde{a}_{20}+\frac{1}{5 \sqrt{7}} \tilde{a}_{30}\right) .
\end{aligned}
$$

We use tilde for $\tilde{a}_{\ell m}$ in Eqs. (7) and (8) because these coefficients are not the same as the conventional $a_{\ell m}$ related to our location and galactic coordinate system. In Fig 1 you can find a schematic representation of multipole components which contribute to the observed CMB frequency spectrum distortions due to scattering.

One can notice from Eqs. (5) and (8) the following features of the formation of spectral distortions due to Compton scattering of anisotropic radiation.

1. The distortion of the form $-x \frac{d B}{d x}$ [term 1 in Eq. (5)] arises as a result of mixing flows with different temperatures due to Thomson scattering. Since $\Theta_{e} \ll 1$, the amplitude of this distortion is dominated by $\beta_{1}$. This coefficient consists of two terms. The first one is the component $\tilde{a}_{20}$ of the local quadrupole temperature anisotropy. It is worth noticing, that this is the only quadrupole component, which does not produce polarization. The second term has the following physical meaning. If there is a hot spot in the CMB anisotropy behind the SZ cluster, then hotter photons, propagating towards the observer through the SZ cluster, are partially scattered out of the line of sight and replaced by cooler photons scattered in the line of sight. As a result of this effect, we would observe a blackbody radiation with a bit lower temperature than in the absence of SZ cluster. Therefore, both effects change the temperature of radiation. Indeed, the term $\frac{\Delta_{T}(\boldsymbol{\Omega})}{T_{r}} x \frac{d B}{d x}$ is in fact the difference between two blackbodies' spectra, $B\left(T_{r}\right)-B\left(T_{r}+\Delta_{T}(\boldsymbol{\Omega})\right)$, in linear approximation. That's why it is rather difficult to use distortion of this kind to find the $\tilde{a}_{20}$ : this effect simply changes the temperature, leaving the spectrum in a blackbody shape.

2. The deviation of the form $\frac{1}{x^{2}} \frac{d}{d x}\left[x^{4} \frac{d}{d x}\left(-x \frac{d B}{d x}\right)\right]$ [term 2 in Eq. (5)] has a very distinctive nonblackbody features. This is the deviation, that we call the
"Anisotropic thermal SZ effect". The amplitude of this deviation is proportional to the linear combination of the local dipole, quadrupole and octupole components only. In Fig. 2 we demonstrate this distortion in comparison with the usual thermal SZ effect. In the next section we show how to use these spectral features in the radiation coming from nearby SZ clusters to independently estimate the $a_{\ell m},(1 \leq \ell \leq 3,-\ell \leq m \leq \ell)$ components, that we directly observe on the sky. We also show how to distinguish between the SW and ISW effects combining the signals from nearby and distant clusters.

\section{THE ESTIMATION OF THE CMB $\ell=1,2,3$ ANISOTROPY MULTIPOLES AND SEPARATION OF SW AND ISW EFFECTS}

In this Section we describe the method to reconstruct the CMB dipole, quadrupole and octupole at our location and show how to separate contributions from the SW and ISW effects to CMB anisotropy by observing the distorted signals from $\mathrm{SZ}$ galaxy clusters. The outline of this section is as follows. In Sec. III A we demonstrate the possibility of $a_{\ell m}$ reconstruction for the simplest possible model and an ideal experiment with no noise. In Sec. III B we consider the $a_{\ell m}$ spatial autocorrelations caused by SW and ISW effects and propose a method for separating SW and ISW contributions to the total $a_{\ell m}$. Finally in Sec. III $\mathrm{C}$ we make our estimates of relative errors for different $\ell$ and $m$ using SZ clusters from the Planck catalog.

\section{A. The possibility of $a_{l m}$ reconstruction}

We start our analysis with a very simple and visual approximation. Let us assume for simplicity, that the CMB incident to all observed SZ clusters has exactly the same anisotropy.

Let us denote by $a_{\ell m}^{j}$ coefficients of the temperature anisotropy decomposition for the observer located at cluster number $j$. Taking into account our simple approximation we consider these coefficients as equal to those we directly observe in the sky: $a_{\ell m}^{j}=a_{\ell m}$. This gives us an opportunity to estimate $a_{\ell m}, \ell=1,2,3,-\ell \leq m \leq \ell$ by observing the $\mathrm{CMB}$ radiation spectral distortions coming from such clusters. The relations between $a_{\ell m}$ of the conventional galactic coordinates and $\tilde{a}_{\ell m}$ defined in Eq. (7) are given by the rotation of the coordinate system. This rotation transforms a spherical harmonic of degree $\ell$ and order $m$ into a linear combination of spherical harmonics of the same degree. Therefore the $\tilde{a}_{\ell 0}^{j}$ coefficients can be expressed in terms of $a_{\ell m}$ as follows:

$$
\tilde{a}_{\ell 0}^{j}=\sum_{m=-\ell}^{\ell} D_{\ell, j}^{0, m} a_{\ell m}^{j} \approx \sum_{m=-\ell}^{\ell} D_{\ell, j}^{0, m} a_{\ell m}
$$


where $D_{\ell, j}^{m, m^{\prime}}$ are the complex conjugate of the Wigner D-matrix elements for the $j$-th cluster. These elements are completely described by the position on the sky of the cluster number $j$. According to Eqs. (5) and (8) the ratio of the amplitude of $\frac{1}{x^{2}} \frac{d}{d x}\left[x^{4} \frac{d}{d x}\left(x \frac{d B}{d x}\right)\right]$ signal to the classical SZ thermal effect is

$$
\begin{aligned}
& s_{j}=-\frac{3}{5 \sqrt{3 \pi}} \sum_{m=-1}^{1} D_{1, j}^{0, m} a_{1 m}+\frac{1}{4 \sqrt{5 \pi}} \sum_{m=-2}^{2} D_{2, j}^{0, m} a_{2 m}- \\
& -\frac{3}{20 \sqrt{7 \pi}} \sum_{m=-3}^{3} D_{3, j}^{0, m} a_{3 m}, \quad j=1, . ., N .
\end{aligned}
$$

In total 15 coefficients $a_{\ell m}$ with $\ell=1,2,3$ and $-\ell \leq m \leq$ $\ell$ contribute to $s_{j}$. This means, that at least $15 \mathrm{SZ}$ objects should be used to solve the linear system (10). Despite the fact that this is a mathematical certainty, in reality the use of 15 clusters only can lead to a completely wrong result because the contribution to the signal from the octupole is overshadowed by the dipole and quadrupole [see Eq. (8), coefficient $\beta_{3}$ ]. This means, that even a small noise can lead to an incorrect reconstruction of $a_{3 m}$. Besides, as we show in the next subsection, there are spatial fluctuations of $a_{\ell m}$ mainly due to the ISW effect. Therefore, even in the case of an ideal experiment we need many more clusters to perform the reconstruction. In Fig. 3 we show the expected signal $s_{j}$ from nearby clusters without the dipole contribution in case where our measurements of the quadrupole and octupole by COBE, WMAP and Planck contain information about the real cosmological signal.

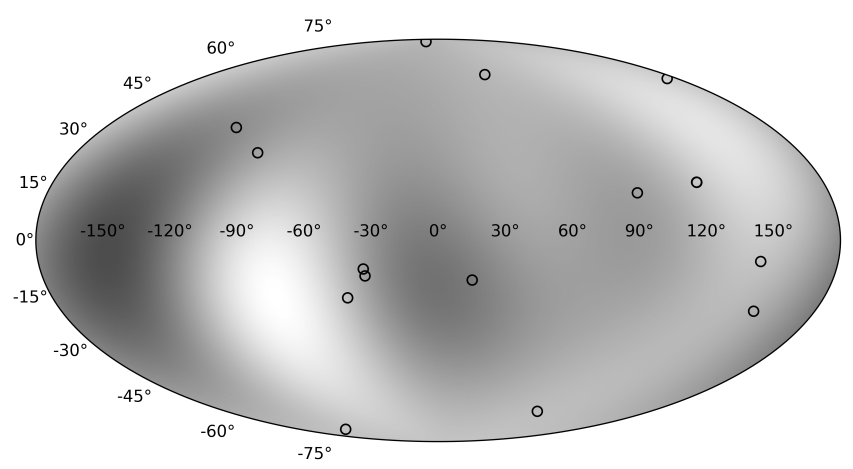

FIG. 3: Map of the expected distribution of the distorted signal on the sky (galactic coordinates) coming from nearby SZ clusters. This map is constructed from the quadrupole and octupole measured by Planck. The unknown dipole is not taken into account. Circles represent 15 nearest clusters from PLANCKSZ2 catalog.

\section{B. ISW and SW effects and $a_{\ell m}$ spatial correlations}

According to Ref. [38, for $\Omega_{\Lambda}=0.7$ on average $40 \%$ of the quadrupole amplitude is generated by the ISW effect, while for the octupole this quantity reaches $25 \%$. The rest is caused by the SW effect on the last scattering surface. In order to estimate the spatial variations of both effects, we simulate a Gaussian distribution of a large scale gravitational potential in a $40 \mathrm{Gpc} / \mathrm{h}$ cubic box with $512^{3}$ grid cells in accordance with the known cosmological power spectrum. To compute the ISW effect, we convolve this field with the kernel taken from Ref. 38 for $\ell=1,2,3$ and $m=0$. Thus we obtain maps of $a_{\ell 0}$ generated by the ISW effect at the same local time everywhere. From these maps we compute the correlation of $a_{\ell 0}$ along the $z$ axis of the box. This is sufficient, since only this projection contributes to the signal we discuss; see Fig. 1. We see distant clusters in their past, i.e., when the Universe was younger and the ISW effect was weaker. To take this into account we multiply the correlation function by the growth factor of the ISW effect. We obtain the correlation function for the SW effect in a similar manner, using a thin spherical layer as a convolution kernel. We do not take into account the variations of the last scattering surface radius with time, since we are interested in small distances, $<2000 \mathrm{Mpc} / \mathrm{h}$ (the present radius of the last scattering surface is $9500 \mathrm{Mpc} / \mathrm{h}$ in the comoving coordinate frame). The obtained correlation functions of the ISW and SW effects are shown in Fig. 4 .

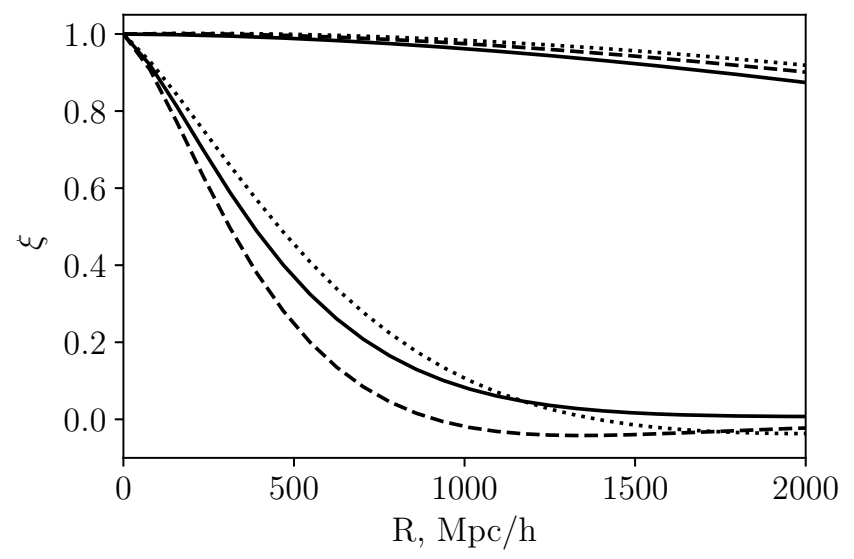

FIG. 4: Correlation functions for dipole (dots), quadrupole (solid) and octupole (dashes) for the SW effect on the last scattering surface (top 3 lines) and the ISW effect (bottom three lines).

As one can see from Fig. 4, the correlation length for the SW effect is much larger than that for the ISW effect. This means that we have three regimes. For the clusters at distances smaller than the ISW correlation length we have $a_{\ell m}$ close to their local values. At distances much larger than the ISW correlation length we will detect $a_{\ell m}$ which have no ISW contribution, since 


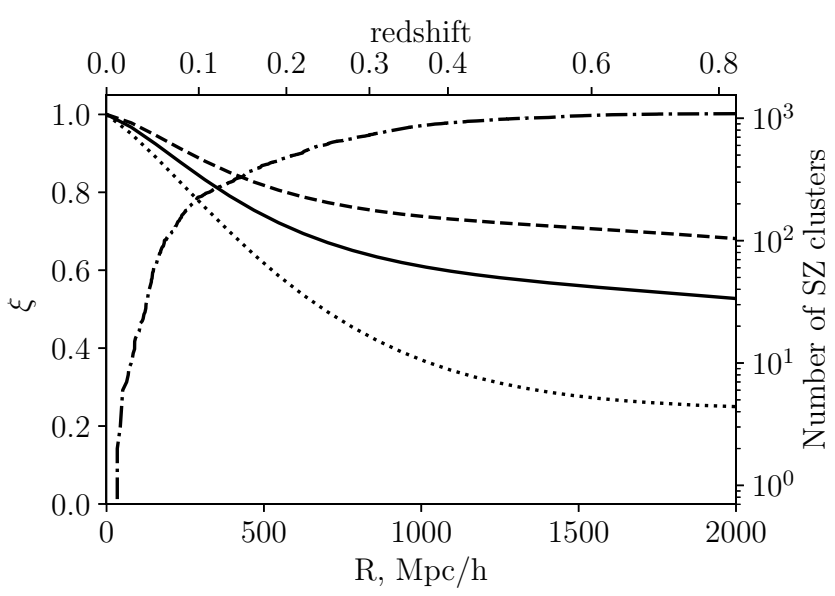

FIG. 5: Total (SW+ISW) correlation functions for dipole (dots), quadrupole (solid) and octupole (dashes). The dotdashed line shows the number of clusters at distance $<R$ from PLANCKSZ2 catalog 39].

we see a much younger Universe. Most SZ clusters are located closer than the SW correlation length, so they will share the same $a_{\ell m}$ 's from the SW effect. At intermediate distances, comparable to the correlation length of the ISW effect, the amplitudes of spherical harmonics will fluctuate. The total correlation function of the $a_{\ell m}$ 's detected by our method is shown in Fig. 5 together with cluster counts. One should note that it describes only the correlation of the local $a_{\ell m}$ with distant ones, not the correlation between two distant points.

One can conclude from this analysis, that we have a unique opportunity to separate ISW and SW effects. Signals from distant clusters contain information about the SW effect only. Therefore dipole, quadrupole and octupole reconstructed by using only distant clusters will contain information about the CMB anisotropy directly at the surface of last scattering. In order to reconstruct total $a_{\ell m}, l=1,2,3$ (SW+ISW effect) in our location we shall use only nearby clusters.

\section{Precision of $a_{\ell m} \ell=1,2,3$ reconstruction}

We estimate the relative precision for the reconstruction of different $a_{\ell m}$ 's using measurements of CMB spectral distortions in the direction of SZ clusters. For simplicity we assume that $a_{\ell m}$ 's are equal at the positions of clusters. This assumption should be valid in two cases, as discussed before: at very small distances, $R<250 \mathrm{Mpc} / \mathrm{h}$ where we can measure the sum of the SW and ISW effects (i.e. $a_{\ell m}$ 's are the same as measured directly from the CMB), or at large distances, $R>1000 \mathrm{Mpc} / \mathrm{h}$, where anisotropy is generated only by the SW effect. Assuming also equal and independent measurement errors of our signal given in Eq. (10), we obtain estimates of the errors of $a_{\ell m}$ measurements using the maximum likelihood method.

In Figs. 6 and 7 we show relative errors of 15 coefficients for 173 clusters at $R<250 \mathrm{Mpc} / \mathrm{h}$ and 225 clusters at $R>1000 \mathrm{Mpc} / \mathrm{h}$. They are normalized by the largest error set to one. We use $4 \pi$ normalized real spherical harmonics for this computation. One can see that amplitudes with $m= \pm \ell$ have the largest errors for every $\ell$. This happens because $Y_{m}^{ \pm m} \sim \sin ^{m}(\theta)$. Most of the power of these harmonics are close to the Galactic plane $(\theta \approx \pi / 2)$. This is the zone of avoidance of Planck SZ catalog, where there are no clusters. In Fig. 8 we show how the relative errors for dipole, quadrupole and octupole powers $\left(C_{1}, C_{2}, C_{3}\right)$ depend on the number of clusters or distance for the sample of $R>1000$ clusters.

From this analysis one can conclude, that to reliably recover all 15 components of the three lowest multipoles the sensitivity should be at least one order of magnitude higher, than that needed for the detection of the total signal.

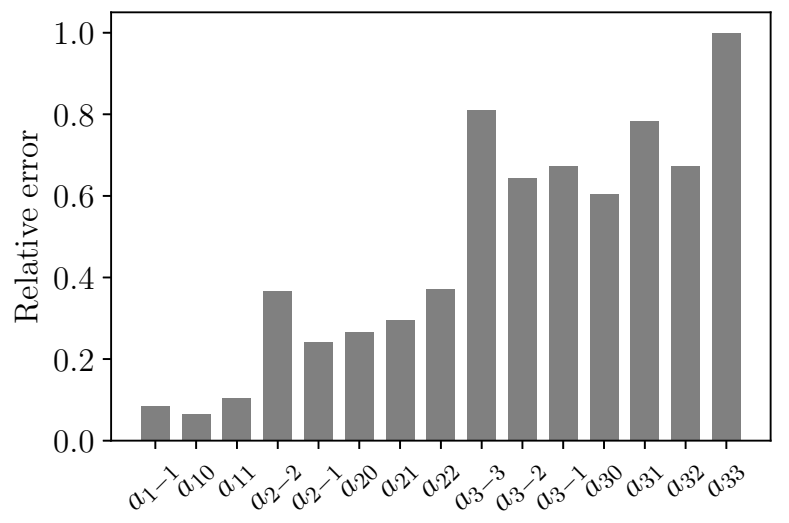

FIG. 6: Relative errors of 15 multipole amplitudes measured from the CMB spectral distortions in SZ clusters at $R<$ $250 \mathrm{Mpc} / \mathrm{h}$.

\section{CONCLUSIONS}

In our paper we derived the correction to the thermal Sunyaev-Zel'dovich effect due to the presence of $\ell=1,2,3$ anisotropy in CMB radiation. The signal corresponding to this correction has a very distinguishable features and can be separated from other nonblackbody components. This signal is strong enough to be detected by upcoming Millimetron mission. We have not considered in details a possible way of components separation in the signal we about to observe to identify the effect, that we described. We only have to mention, that in order to isolate the effect considered by us, it is necessary to take into account relativistic corrections to the isotropic thermal SZ effect at least up to the fifth order, the kinematic SZ effect and corrections to it, as well as multiple scattering. In other words we should consider 


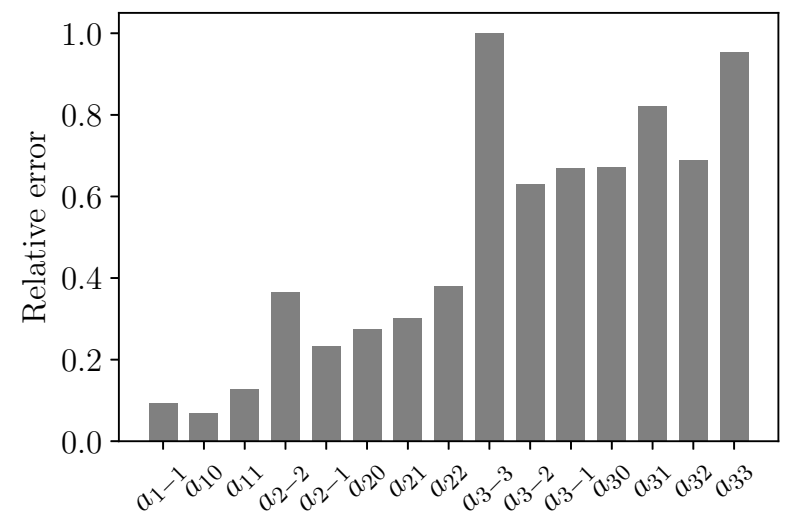

FIG. 7: Relative errors of 15 multipole amplitudes measured from the CMB spectral distortions in SZ clusters at $R>$ $1000 \mathrm{Mpc} / \mathrm{h}$.

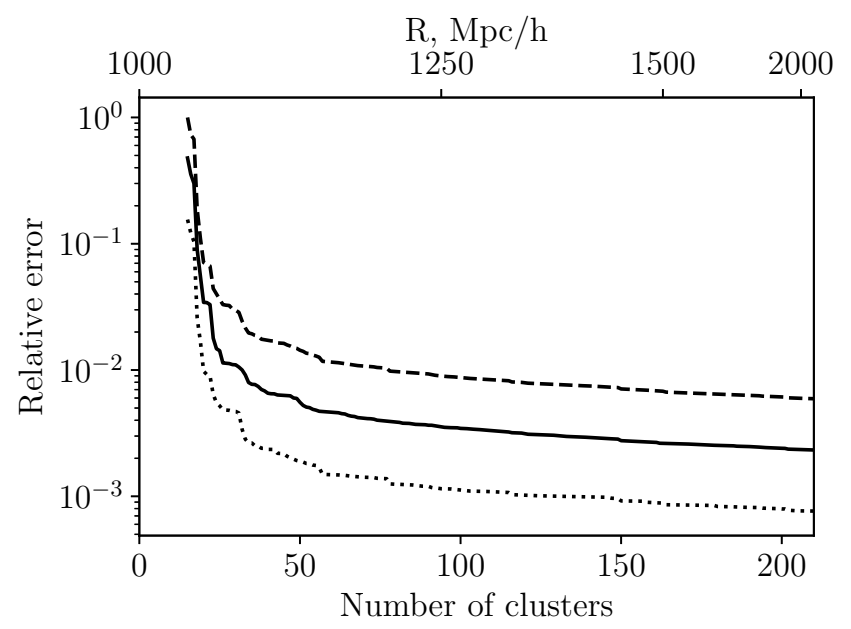

FIG. 8: Relative error of the power of dipole (dots), quadrupole (solid) and octupole (dashed) for a sample of distant clusters as a function of distance or number of clusters (starting from 15 clusters). all effects of the same and higher orders. This correction makes it possible to independently estimate low the CMB anisotropy multipoles by observing the spectral distortions from galaxy clusters. We can use both nearby and distant clusters. The nearby clusters can be used for independent estimations of all three local multipolesdipole, quadrupole and octupole -in our location. As for the distant clusters, they can be used to measure the signal directly from the surface of last scattering without the ISW contribution. Therefore the anisotropic thermal SZ effect gives us an opportunity to separate the ISW and SW effects.
We wish to thank the Referee for very helpful discussion. The work is supported by the Program 28 of the fundamental research of the Presidium of the Russian Academy of Sciences "Space: research of the fundamental processes and relations", subprogram II "Astrophysical objects as space laboratories" and by the Project 01-2018 of LPI new scientific groups.
[1] A. S. Kompaneets, Soviet Journal of Experimental and Theoretical Physics 4, 730 (1957).

[2] Y. B. Zeldovich and R. A. Sunyaev, Astrophys. Space. Sci 4, 301 (1969).

[3] R. A. Sunyaev and Y. B. Zeldovich, Astrophys. Space. Sci 7, 20 (1970).

[4] A. Challinor and A. Lasenby, Astrophys. J 499, 1 (1998), astro-ph/9711161.

[5] N. Itoh, Y. Kohyama, and S. Nozawa, Astrophys. J 502, 7 (1998), astro-ph/9712289.

[6] A. Stebbins, Submitted to: Astrophys. J. Lett. (1997), astro-ph/9709065.

[7] M. Shimon and Y. Rephaeli, Astrophys. J. 575, 12 (2002), astro-ph/0204355.

[8] N. Itoh, Y. Kawana, S. Nozawa, and Y. Kohyama, ArXiv
Astrophysics e-prints (2000), astro-ph/0005390.

[9] A. D. Hincks, R. Génova-Santos, G. Luzzi, and E. S. Battistelli, ArXiv e-prints arXiv:1803.03277 (2018), 1803.03277.

[10] S. Nozawa, N. Itoh, and Y. Kohyama, Astrophys. J 508, 17 (1998), astro-ph/9804051.

[11] S. Y. Sazonov and R. A. Sunyaev, Astrophys. J 508, 1 (1998).

[12] A. Challinor and A. Lasenby, Astrophys. J 510, 930 (1999), astro-ph/9805329.

[13] N. Itoh, S. Nozawa, and Y. Kohyama, Astrophys. J 533, 588 (2000), astro-ph/9812376.

[14] J. Chluba, D. Nagai, S. Sazonov, and K. Nelson, Mon. Not. R. Astron. Soc 426, 510 (2012), 1205.5778.

[15] S. Nozawa and Y. Kohyama, Phys. Rev. D 79, 083005 
(2009), 0902.2595

[16] S. Nozawa and Y. Kohyama, Mon. Not. R. Astron. Soc 434, 710 (2013), 1303.2286.

[17] S. Nozawa and Y. Kohyama, Mon. Not. R. Astron. Soc 441, 3018 (2014), 1402.1541.

[18] S. Yasini and E. Pierpaoli, Phys. Rev. D 94, 023513 (2016).

[19] S. A. Balashev, E. E. Kholupenko, J. Chluba, A. V. Ivanchik, and D. A. Varshalovich, Astrophys. J. 810, 131 (2015), 1505.06028.

[20] S. Yasini and E. Pierpaoli, Physical Review Letters 119, 221102 (2017), 1610.00015.

[21] J. Chluba, R. Khatri, and R. A. Sunyaev, Mon. Not. R. Astron. Soc 425, 1129 (2012), 1202.0057.

[22] J. Chluba, L. Dai, and M. Kamionkowski, Mon. Not. R. Astron. Soc 437, 67 (2014), 1308.5969.

[23] J. Chluba and L. Dai, Mon. Not. R. Astron. Soc 438, 1324 (2014), 1309.3274.

[24] J. P. Babuel-Peyrissac and G. Rouvillois, Journal de Physique 30, 301 (1969).

[25] G. Efstathiou, Mon. Not. R. Astron. Soc 346, L26 (2003), astro-ph/0306431.

[26] M. Tegmark, A. de Oliveira-Costa, and A. J. Hamilton, Phys. Rev. D 68, 123523 (2003), astro-ph/0302496.

[27] D. J. Schwarz, G. D. Starkman, D. Huterer, and C. J. Copi, Physical Review Letters 93, 221301 (2004), astroph/0403353.

[28] Planck Collaboration, P. A. R. Ade, N. Aghanim, C. Armitage-Caplan, M. Arnaud, M. Ashdown, F. AtrioBarandela, J. Aumont, C. Baccigalupi, A. J. Banday, et al., Astron. Astrophys 571, A15 (2014), 1303.5075.

[29] C. J. Copi, D. Huterer, and G. D. Starkman, Phys. Rev. D 70, 043515 (2004), astro-ph/0310511.

[30] C. J. Copi, D. Huterer, D. J. Schwarz, and G. D. Stark- man, Mon. Not. R. Astron. Soc 367, 79 (2006), astroph/0508047.

[31] P. D. Naselsky and O. V. Verkhodanov, International Journal of Modern Physics D 17, 179 (2008), astro$\mathrm{ph} / 0609409$.

[32] R. K. Sachs and A. M. Wolfe, Astrophys. J 147, 73 (1967).

[33] W. Wild, N. S. Kardashev, S. F. Likhachev, N. G. Babakin, V. Y. Arkhipov, I. S. Vinogradov, V. V. Andreyanov, S. D. Fedorchuk, N. V. Myshonkova, Y. A. Alexsandrov, et al., Experimental Astronomy 23, 221 (2009).

[34] N. S. Kardashev, I. D. Novikov, V. N. Lukash, S. V. Pilipenko, E. V. Mikheeva, D. V. Bisikalo, D. S. Wiebe, A. G. Doroshkevich, A. V. Zasov, I. I. Zinchenko, et al., Physics Uspekhi 57, 1199-1228 (2014), 1502.06071.

[35] A. V. Smirnov, A. M. Baryshev, S. V. Pilipenko, N. V. Myshonkova, V. B. Bulanov, M. Y. Arkhipov, I. S. Vinogradov, S. F. Likhachev, and N. S. Kardashev, in Space Telescopes and Instrumentation 2012: Optical, Infrared, and Millimeter Wave (2012), vol. 8442 of Proc. SPIE, p. 84424C.

[36] V. Desjacques, J. Chluba, J. Silk, F. de Bernardis, and O. Doré, Mon. Not. R. Astron. Soc 451, 4460 (2015), 1503.05589 .

[37] J. Chluba, ArXiv e-prints arXiv:1405.6938 (2014), 1405.6938.

[38] R. G. Crittenden and N. Turok, Physical Review Letters 76, 575 (1996), astro-ph/9510072.

[39] Planck Collaboration, P. A. R. Ade, N. Aghanim, M. Arnaud, M. Ashdown, J. Aumont, C. Baccigalupi, A. J. Banday, R. B. Barreiro, R. Barrena, et al., Astron. Astrophys 594, A27 (2016), 1502.01598. 